Supporting Information

\title{
Tunable Electrical-Sensing Performance of Random-Alternating Layered Graphene/Polyaniline Nanoarchitectures
}

Min-Sik Kim, ${ }^{2}$ Saerona Kim, ${ }^{2}$ Hye Jeong Kong, ${ }^{2}$ Oh Seok Kwon, ${ }^{3}$ and Hyeonseok Yoon ${ }^{1,2, *}$

${ }^{1}$ School of Polymer Science and Engineering, Chonnam National University, 77 Yongbong-ro,

Buk-gu, Gwangju 61186, South Korea. E-mail: hyoon@chonnam.ac.kr; Fax: +82-62-530-1779; Tel: +82-62-530-1778

${ }^{2}$ Department of Polymer Engineering, Graduate School, Chonnam National University, 77 Yongbong-ro, Buk-gu, Gwangju 61186, South Korea.

${ }^{3}$ BioNanotechnology Research Center, Korea Research Institute of Bioscience and

Biotechnology (KRIBB), 125 Gwahak-ro, Yuseong-gu, Daejeon 34141, South Korea

\section{AUTHOR INFORMATION}

\section{Corresponding Authors}

*Hyeonseok Yoon, E-mail: hyoon@chonnam.ac.kr 

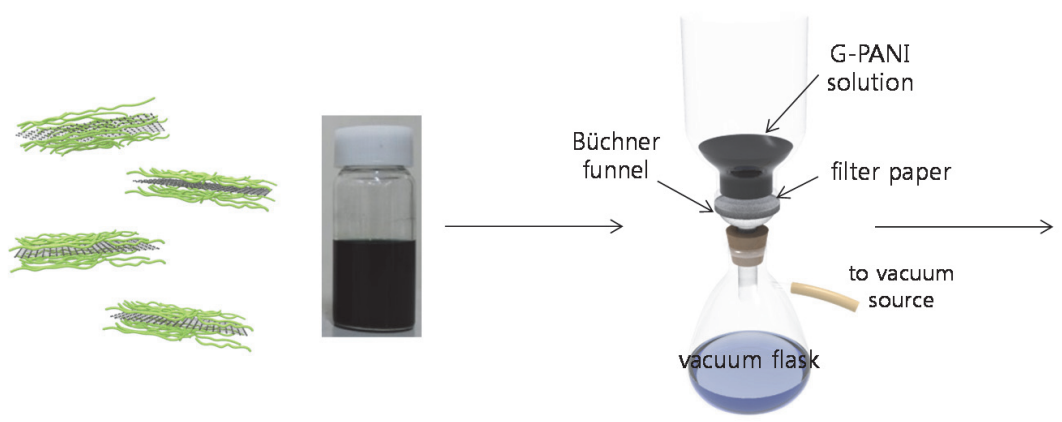

G-PANI solution

Graphene-to-PANI weight ratio 1:2
Filtration

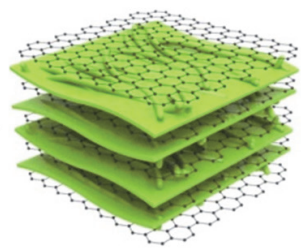

G-PANI film

Figure S1. Scheme describing the overall process of preparing G-PANI film. 

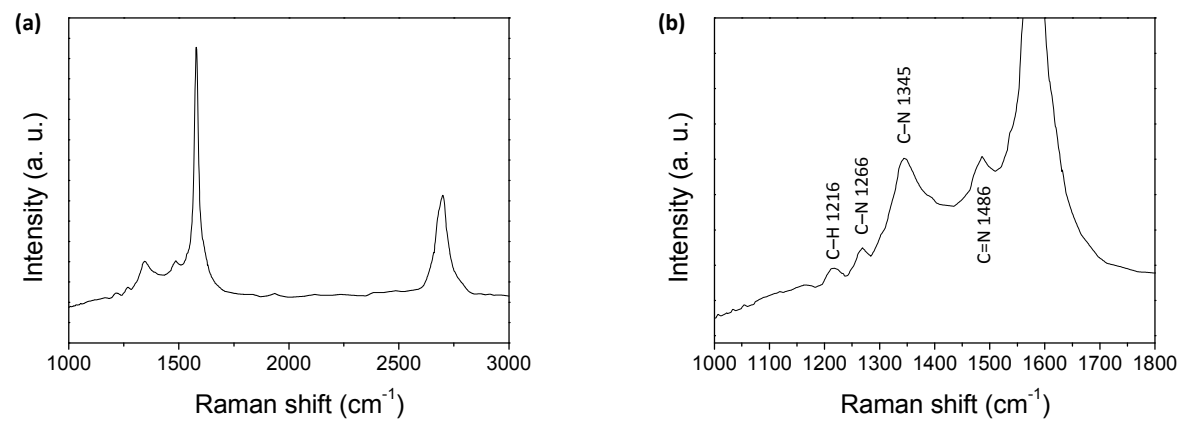

Figure S2. A representative Raman spectrum of G-PANI nanoarchitectures: (a) 1000 to 3000 $\mathrm{cm}^{-1}$; (b) 1000 to $1800 \mathrm{~cm}^{-1}$. 


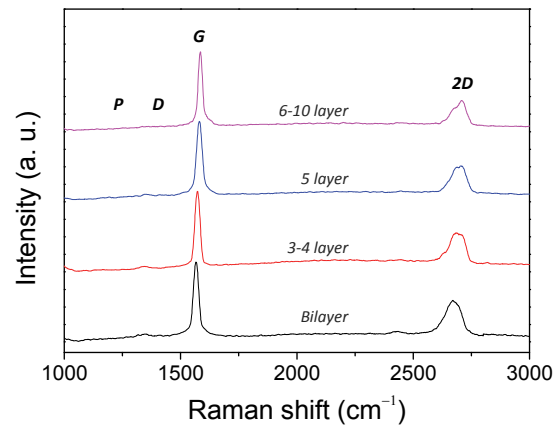

Figure S3. Raman spectroscopy analysis of graphenes exfoliated by PANI.

Raman spectroscopy analysis was further performed to examine the layer number of exfoliated graphenes in G-PANI nanoarchitectures. The G-PANI solution was deposited on a Si wafer and washed several times using NMP to partly remove the surface PANI. 

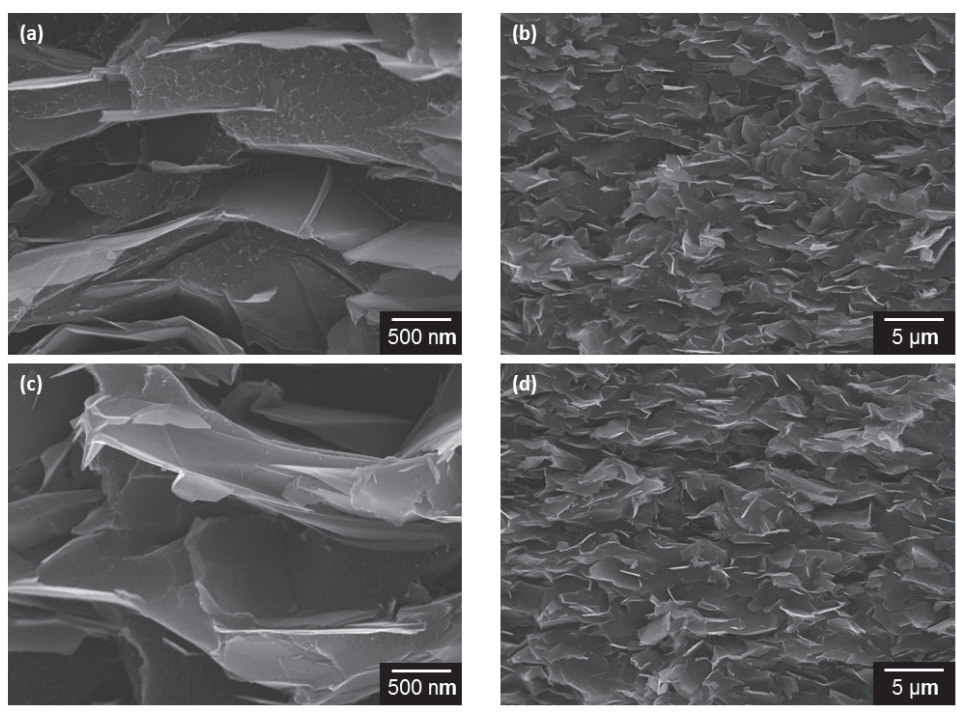

Figure S4. SEM images of G-PANI nanoarchitectures doped with $(a, b)$ CSA/DBSA and $(c, d)$ CSA/SBT. 


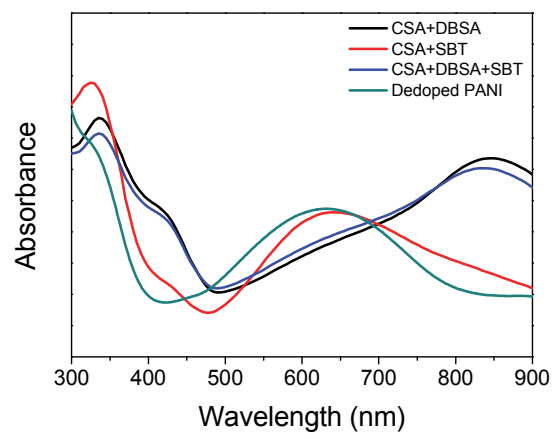

Figure S5. UV-visible absorption spectra of PANIs treated with different dopants.

UV-visible spectroscopy analysis provides the effect of dopants on the oxidation level of PANI. The absorption bands around 360,430 , and $820 \mathrm{~nm}$ arise from $\pi-\pi^{*}$, polaron- $-\pi^{*}$, and $\pi-$ polaron electronic transitions in emeraldine salt PANI, and the absorption bands around 330 and $630 \mathrm{~nm}$ are due to $\pi-\pi^{*}$ and exciton transitions in emeraldine-based PANI. As a result, it was found that CSA and DBSA dopants significantly contributed to the oxidation level of PANI. 


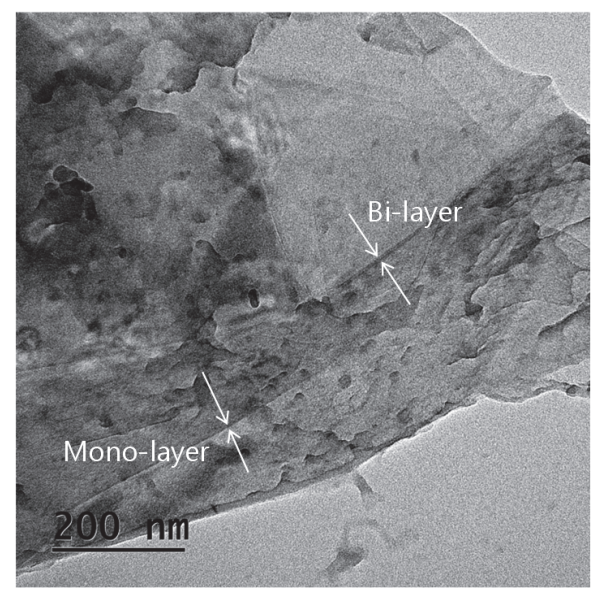

Figure S6. A typical HRTEM image of G-PANI nanoarchitectures, in which mono-layer and bilayer graphenes were marked with the arrows. For TEM observation, a drop of diluted G-PANI solution was deposited on a copper grid. 

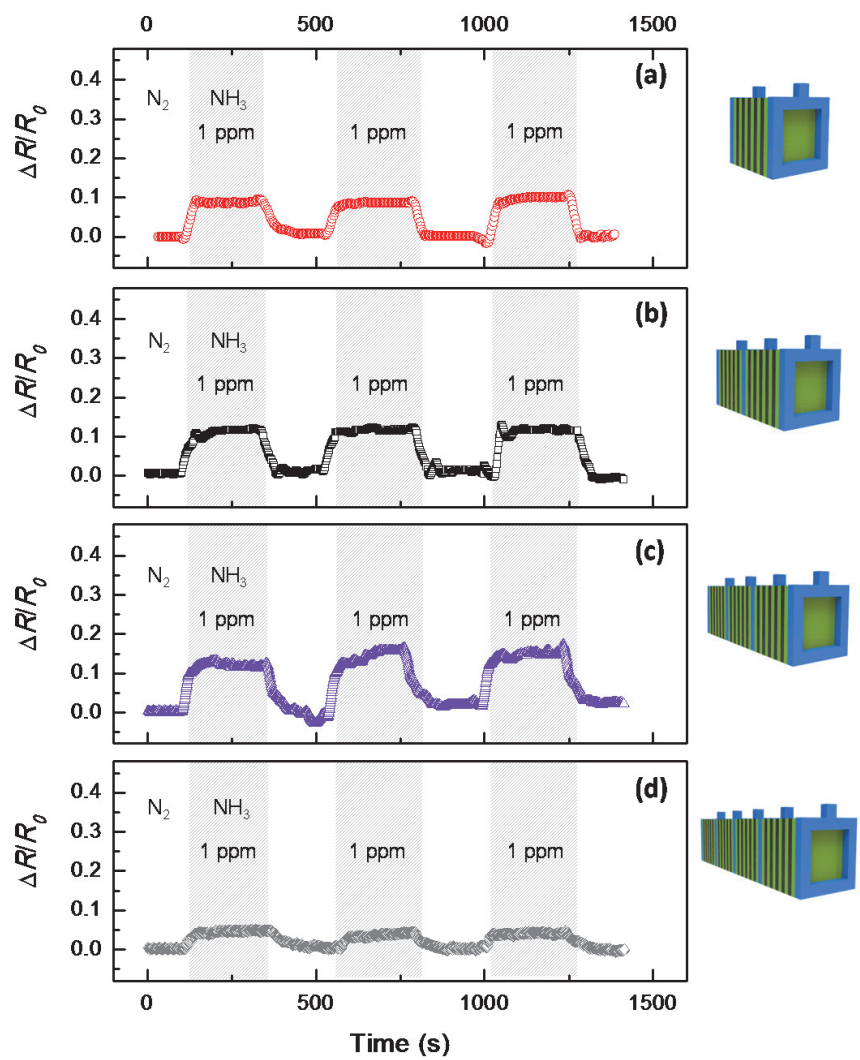

Figure S7. Electrical responses of G-PANI electrodes upon periodic exposure to $1 \mathrm{ppm}$ ammonia, in which the thickness of the G-PANI film inserted between the electrodes was adjusted: (a) $T$, (b) $2 T$, (c) $3 T$, (d) $4 T$. 